\title{
A case of intradural lumbar disc herniation
}

\author{
Utsav Bhattarai $^{1}$, Pritam Gurung ${ }^{1}$, Janam Shrestha ${ }^{1}$, Sudan Dhakal ${ }^{1}$, Samir Acharya ${ }^{2}$, and \\ Basant Pant ${ }^{2}$ \\ ${ }^{1}$ Annapurna Neurological Institute and Allied Sciences \\ ${ }^{2}$ Annapurna Neurological Institute and allied Sciences
}

October 17, 2021

\begin{abstract}
Lumbar disc herniation into the dural space is a rare phenomenon of degenerative lumbar disc disease and its pathogenesis remains unclear. Intraoperative ultrasonography and histopathological examination of resected specimen aids in diagnosis of intradural disc herniation.Prompt surgery is recommended as postoperative outcome of the patient at our setting was favourable.
\end{abstract}

\section{INTRODUCTION}

Lumbar disc herniation into the dural space is a very rare phenomenon of degenerative lumbar disc disease pertaining uncertain pathology with most common region involvement being L4- L5 and L5-S1 level.[1]. Magnetic resonance imaging (MRI) remains the best tool in diagnosis of this disease entity however preoperative diagnosis still remains a difficult task. In this report, we describe a patient who underwent surgical resection of a intradural disc.

\section{CASE PRESENTATION}

A 43-year-old man who presented at our emergency room (ER) with complain of left sided lower back pain radiating to left lower limb for 2 years with increased severity since last 10 days, weakness in his left lower limb since 10 days and with history of urinary incontinence (overflow) since last 2 days. General physical examination revealed no abnormality. On straight leg raising test both legs was found to be 60 degree on the right leg and 50 degree on the left leg, extensor hallucis longus (EHL) and flexor hallucis longus (FHL) was $3 / 5$ on both sides and sensory examination showed reduced sensation on S1 dermatome on the right. MRI of the lumbo sacral spine revealed diffuse bulging of disc with the left postero central / sub articular extrusion at L5-S1 level, thecal compression and impingement of the left traversing S1 nerve root (Figure 1 $\mathrm{A}, \mathrm{B}$ and $\mathrm{C}$ ). Presumptive diagnosis of herniated lumbar disc with radiculopathy and cauda equina syndrome was made at ER on the basis of clinical evaluation of patient.

Patient underwent L5 laminectomy. Disc was removed from both side from shoulder end of nerve root. Foraminotomy was performed bilaterally. Intraoperatively only minimal disc fragments was found over epidural space. The dorsal dura matter was tense and bulging at L5-S1 level (Figure 2). Subsequently, dorsal durotomy was performed upon which revealed hard fungating mass similar to tissue texture of nucleus pulposus over the arachnoid membrane ( Figure 3). The mass was dissociative and revelaed itself upon durotomy. Histology report confirmed it to be disc tisue.

Post operatively, patient's left lower back pain and left leg radiculopathic pain along with left lower limb weakness improved significantly.

\section{DISCUSSION}


Intradural disc herniation (IDH) is a rare phenomenon, which accounts for only 0.25 to $0.35 \%[2]$ of all disc herniations with lumbar region being the most common site of occurrence[3]. The level most frequently affected is L4-L5 [4]. Male are more likely to have the disease entity than female. Average age of onset is between 50 -60 yrs. Symtoms of IDH may vary depending upon location of disc herniation, with cervical IDH patients presenting with clinical findings of Brown Sequard Syndrome [5], [6], or incomplete and trainsient quadriparesis and those involving lumbar region presenting as long history of radiculopathic pain with acute presentation consisting of symptoms of cauda equina syndrome [4]. In our patient both radiculopathy and cauda equina symptoms improved gradually post operatively with poor improvement of bladder function for which physiotherapy and acupuncture therapy was provided to patient.

The pathogenesis of IDH remains unclear, however few theories have been proposed. Several reports suggested that certain factors like reduced epidural space resulting from congenital or iatrogenic narrowing of spinal canal, adhesions between annulus fibrosus, posterior longitudinal ligament (PLL) and ventral dura matter were responsible for IDH [7], [8]. Among the aformentiond pathogenesis adhesion between PLL and ventral dura matter was considered to be an important factor [9], [10]. Tateiwa [11] stated that factors such as local trauma, previous spine surgery or even congenital factors like dural thickness were responsible for adhesions. Floeth and Herdmann [12] reported that chronic inflammation, as a result of degenerative disc pathologies, favors development of adhesions and lead to erosion process with thinning of the dura. Dura matter and PLL are in closest proximity at L4-L5 level which explains the highest incidence of IDH at L4-L5 level [13]. In our case, there was dense adhesion between PLL and ventral dura and we speculate that previous history of trauma with disc degeneration resulted into chronic inflammation, and during this process long term irritation, inflammation with a sudden force caused the prolapsed disc into the intradural space through the dural erosion.

A preoperative assumption and diagnosis of IDH is difficult. Lesions might be ignored or can easily be mistaken for other findings on imaging. Definite diagnosis are made only on surgery, not via imaging alone. The MRI in our case did not indicate the presence of intradural disc fragments and definitive diagnosis was made intraoperatively.

\section{CONCLSUIONS}

A high degree of suspicion of dural disc herniation is raised when intraoperative findings and preoperative image description becomes incompatible. Intraoperative ultrasonography and histopathological examination of resected specimen further aids in diagnosis of dural disc herniation. Prompt surgery is recommended as postoperative outcome of the patient at our setting was favourable.

\section{CONFLICTS OF INTEREST}

None of the authors have potential conflicts of interest to be disclosed.

\section{AUTHOR CONTRIBUTIONS}

Utsav Bhattarai involved in original draft, conceptualization, review, and editing. Pritam Gurung involved in review, and editing. Janam Shrestha involved in review, and editing. Sudan Dhakal and Samir Acharya established the diagnosis and treated the patient. Basant Pant involved in supervision.

\section{ETHICS APPROVAL AND CONSENT TO PARTICIPATE}

Ethical approval of case report is not needed in accordance with the local ethical guideline. Written informed consent was obtained from the patient to include the clinical details.

\section{KEY CLINICAL MESSAGE}

MRI remains the best tool in diagnosis of this disease entity however preoperative diagnosis still remains a difficult task. A high degree of suspicion is raised when intraoperative findings and preoperative image description becomes incompatible.

\section{References}


[1] G. Schisano, A. Franco, and P. Nina, "Intraradicular and intradural lumbar disc herniation: Experiences with nine cases," Surg. Neurol. , vol. 44, no. 6, pp. 536-543, 1995.

[2] H. S. Kim, J. P. Eun, and J. S. Park, "Intradural migration of a sequestrated lumbar disc fragment masquerading as a spinal intradural tumor," J. Korean Neurosurg. Soc. , 2012.

[3] A. Öztürk, E. Avci, P. Yazgan, F. Torun, Ş. Yücetaş, and H. Karabaé, "Intraradural herniation of intervertebral disc at the level of lumbar 1-lumbar 2," Turk. Neurosurg. , 2007.

[4] C. C. Liu, C. T. Huang, C. M. Lin, and K. N. Liu, "Intradural disc herniation at L5 level mimicking an intradural spinal tumor," Eur. Spine J. , 2011.

[5] Q. Guan, F. Xing, Y. Long, and Z. Xiang, "Cervical intradural disc herniation: A systematic review," Journal of Clinical Neuroscience . 2018.

[6] A. Gunasekaran, N. K. M. de los Reyes, J. Walters, and N. Kazemi, "Clinical Presentation, Diagnosis, and Surgical Treatment of Spontaneous Cervical Intradural Disc Herniations: A Review of the Literature," World Neurosurgery . 2018.

[7] S. K. Jain, I. V. Sundar, V. Sharma, R. S. Goel, and R. Gupta, "Intradural disc herniation - A case report," Turk. Neurosurg. , 2013.

[8] H. A. Huliyappa et al. , "Transdural herniated lumbar disc disease with muscle patch for closure of durotomy - A Brief review of literature," Neurologia i Neurochirurgia Polska . 2017.

[9] P. Sarliève, E. Delabrousse, C. Clair, H. Haj Hussein, C. Schmitt, and B. Kastler, "Intradural disc herniation with cranial migration of an excluded fragment," Clin. Imaging , 2004.

[10] K. L. Krajewski and J. Regelsberger, "Intradural lumbar disc herniation associated with degenerative spine disease and rheumatoid arthritis," Spine (Phila. Pa. 1976)., 2013.

[11] D. Tateiwa et al. , "Intradural Disk Herniation Mimicking a Spinal Tumor: Radiologic Imaging, Pathogenesis, and Operative Management," Case Rep. Orthop. , 2018.

[12] F. Floeth and J. Herdmann, "Chronic dura erosion and intradural lumbar disc herniation: CT and MR imaging and intraoperative photographs of a transdural sequestrectomy.," Eur. Spine J. , 2012.

[13] J. Pan et al. , "Intradural cervical disc herniation: Report of two cases and review of the literature," Spine . 2011.

\section{Figure legends}

\section{Figure 1}

MRI lumbar T1 \& T2 axial and T2 saggital revealed focal posterocentral disc bulge with central and B/l paracentral extrusion of the disc elements in the superior and inferior spinal canal at L5-S1 level causing severe narrowing of the central spinal canal and compression of the descending nerve roots and transversing nerve left S1 nerve root.

Figure 2

Intraoperative image showing tense and bulging mass inside the thecal sac at L5-S1 level.

Figure 3

After removal it revealed hard fungating mass similar to tissue texture of nucleus pulposus. 

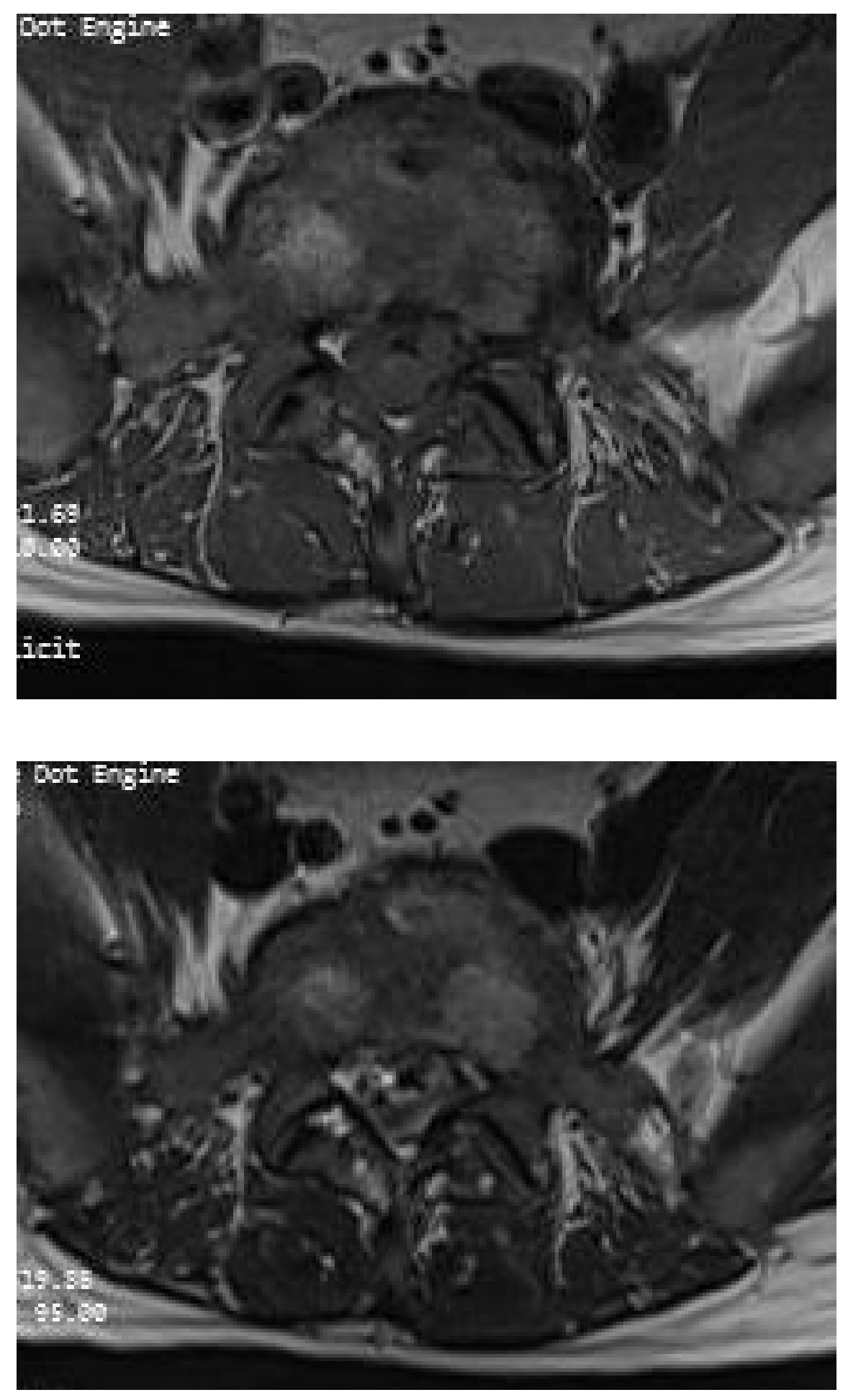


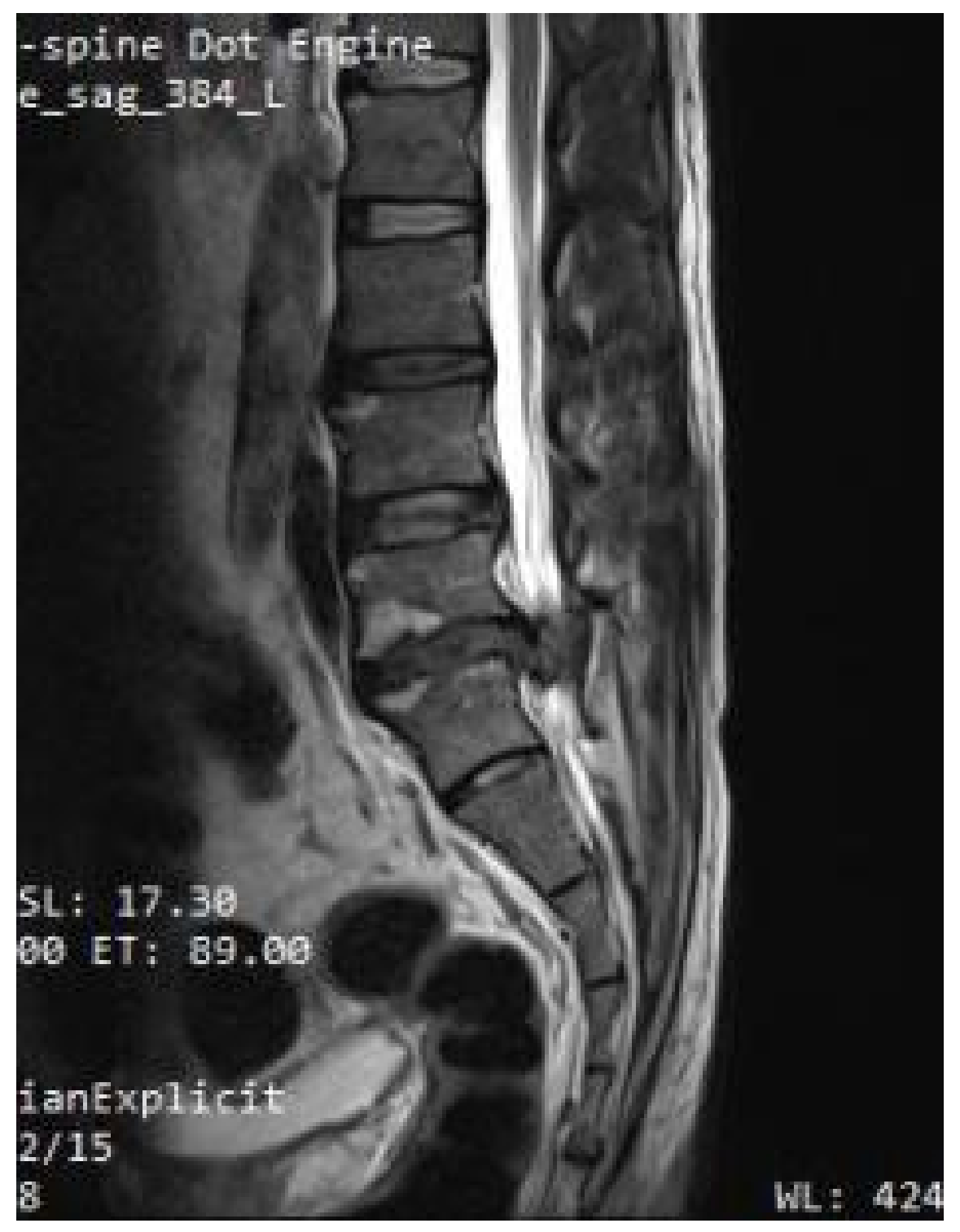



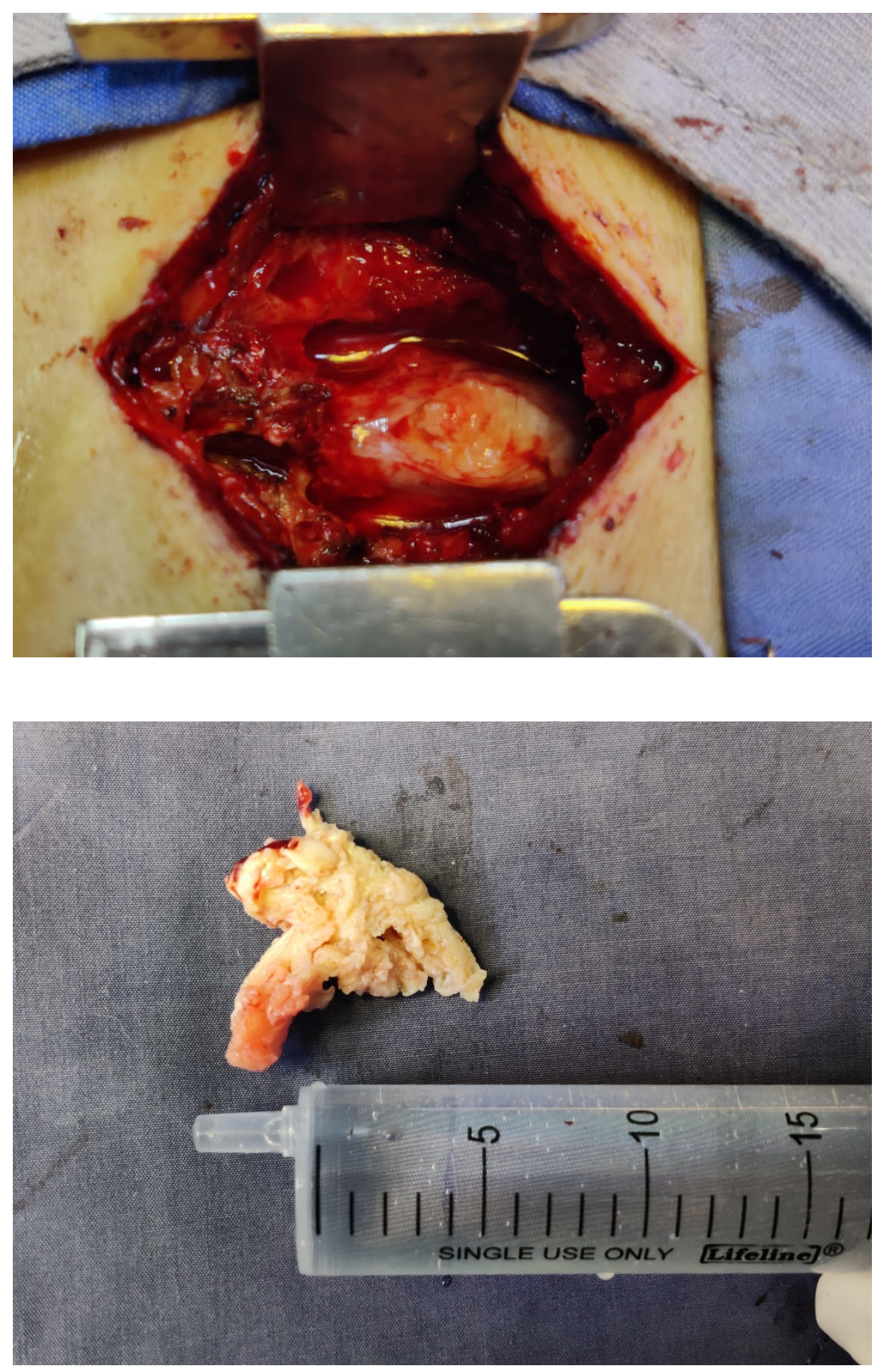